Review Article

\title{
Association between Air Pollution and the Development of Rheumatic Disease: A Systematic Review
}

\author{
Gavin Sun, ${ }^{1}$ Glen Hazlewood, ${ }^{1}$ Sasha Bernatsky, ${ }^{2}$ Gilaad G. Kaplan, ${ }^{1,3}$ \\ Bertus Eksteen, ${ }^{1}$ and Cheryl Barnabe ${ }^{1,3}$ \\ ${ }^{1}$ Department of Medicine, Cumming School of Medicine, University of Calgary, Calgary, AB, Canada \\ ${ }^{2}$ McGill University, Montréal, QC, Canada \\ ${ }^{3}$ Department of Community Health Sciences, Cumming School of Medicine, University of Calgary, Calgary, AB, Canada
}

Correspondence should be addressed to Cheryl Barnabe; ccbarnab@ucalgary.ca

Received 7 July 2016; Revised 15 September 2016; Accepted 4 October 2016

Academic Editor: Lisa Rider

Copyright (C) 2016 Gavin Sun et al. This is an open access article distributed under the Creative Commons Attribution License, which permits unrestricted use, distribution, and reproduction in any medium, provided the original work is properly cited.

\begin{abstract}
Objective. Environmental risk factors, such as air pollution, have been studied in relation to the risk of development of rheumatic diseases. We performed a systematic literature review to summarize the existing knowledge. Methods. MEDLINE (1946 to September 2016) and EMBASE (1980 to 2016, week 37) databases were searched using MeSH terms and keywords to identify cohort, case-control, and case cross-over studies reporting risk estimates for the development of select rheumatic diseases in relation to exposure of measured air pollutants $(n=8)$. We extracted information on the population sample and study period, method of case and exposure determination, and the estimate of association. Results. There was no consistent evidence of an increased risk for the development of rheumatoid arthritis (RA) with exposure to $\mathrm{NO}_{2}, \mathrm{SO}_{2}, \mathrm{PM}_{2.5}$, or $\mathrm{PM}_{10}$. Case-control studies in systemic autoimmune rheumatic diseases (SARDs) indicated higher odds of diagnosis with increasing $\mathrm{PM}_{2.5}$ exposure, as well as an increased relative risk for juvenile idiopathic arthritis (JIA) in American children $<5.5$ years of age. There was no association with SARDs and $\mathrm{NO}_{2}$ exposure. Conclusion. There is evidence for a possible association between air pollutant exposures and the development of SARDs and JIA, but relationships with other rheumatic diseases are less clear.
\end{abstract}

\section{Introduction}

Environmental exposures and genetic predisposition are hypothesized to interact to result in the expression of autoimmune rheumatic diseases such as rheumatoid arthritis (RA), juvenile idiopathic arthritis (JIA), and systematic autoimmune rheumatic diseases (SARDs) [1], as well as other immunemediated diseases such as inflammatory bowel disease [2] and multiple sclerosis [3]. Identifying modifiable risk factors for disease development and prognosis is important to reduce the substantial impact and burden of these chronic diseases in society.

Air pollution is a plausible risk factor for autoimmune disease development. Other inhalants such as tobacco smoke and silica are strongly associated with the development of RA, related to their ability to directly interact with alveolar tissue $[4,5]$. Air pollution has been demonstrated to be able to directly stimulate an inflammatory response [6] and indirectly alter the microbiome [7]. A relationship between particulate matter exposure and elevations in inflammatory marker levels has been described [8-11]. As randomized controlled trials to assess directly for causation between air pollutant exposures and disease development in humans are not feasible, we must rely on observational studies to assess for evidence of associations. Fortunately, several methods to estimate air pollutant exposure exist. A variety of air pollutants from industrial and private sources are measurable at fixedsite continuous monitoring stations that collect hourly mean levels of criteria air pollutants, including particulate matter $<2.5 \mu \mathrm{m}$ in size $\left(\mathrm{PM}_{2.5}\right)$, particulate matter $<10 \mu \mathrm{m}$ in size $\left(\mathrm{PM}_{10}\right)$, sulfur dioxide $\left(\mathrm{SO}_{2}\right)$, nitrogen dioxide $\left(\mathrm{NO}_{2}\right)$, carbon monoxide (CO), and ozone $\left(\mathrm{O}_{3}\right)$. The hourly data can then be averaged to obtain defined temporal estimates for the region (e.g., a city). Land use regression models use Geographic Information System (GIS) to estimate air pollutant exposure 
through a combination of land use, traffic, population density, physical geography, and meteorology across an area [12] and predict concentrations at a defined site, such as an individual's location of residence determined by postal code [13]. Inverse distance weighting (IDW) uses the general principle of interpolation, where values at a site are estimated based on distance from a measured value at another point (e.g., a monitoring station) under the presumption of reducing pollutant levels with increasing distance [14]. Finally, remote sensing through satellite imaging yields estimates across broad geographic areas.

Our objective was to identify studies estimating associations between exposure to the air pollutants listed above and the risk of development of select rheumatic diseases. This included inflammatory arthritis conditions such as RA and JIA, as well as SARDs and individual diseases of autoimmune myositis, systemic lupus erythematosus (SLE), scleroderma, and vasculitis.

\section{Methods}

2.1. Search Strategy. MEDLINE (1946 to September 2016) and EMBASE (1980 to 2016, week 37) databases were searched using MeSH terms and keywords for rheumatic diseases (RA, SLE, JIA, inflammatory myositis, scleroderma, vasculitis, and SARDs) in relation to exposure to measured air pollutants [15] (Search Strategy in Appendix).

2.2. Study Selection. Three authors (Gavin Sun, Glen Hazlewood, and Cheryl Barnabe) independently completed title and abstract and full-text reviews. Studies were included based on the following criteria: assessing the outcome of a rheumatic disease of interest (RA, JIA, SARDs, and individual diseases of autoimmune myositis, SLE, scleroderma, and vasculitis), individual exposure to ambient air pollutants $\left(\mathrm{PM}_{2.5}, \mathrm{PM}_{10}, \mathrm{SO}_{2}, \mathrm{NO}_{2}, \mathrm{CO}\right.$, and $\left.\mathrm{O}_{3}\right)$, and having a casecontrol, case cross-over, or cohort design. Only English language studies were included. The study had to report risk estimates (any of relative risk (RR), hazard ratio (HR), or odds ratio (OR)) with the corresponding 95\% confidence intervals $(95 \% \mathrm{CI})$ or sufficient data for calculation. Reviews, case reports, mechanism studies, and nonhuman studies were excluded.

2.3. Data Extraction and Assessment of Study Quality. Data extraction was performed in duplicate by two authors (Gavin Sun and Cheryl Barnabe). A standard reporting form was developed to extract pertinent information from each study, including the country or region of study, calendar years of study, diagnosis criteria for the rheumatic disease assessed, and the number of patients in case or control groups in each category. The study design and method of assessing air pollutant levels were also extracted. The estimates and their margin of error were extracted. The Newcastle-Ottawa scale [16] was used to assess the quality of the studies relevant to the objective, again in duplicate by two authors (Gavin Sun and Cheryl Barnabe). For case-control studies, quality was assessed for four domains of selection (case definition, representativeness of cases, selection of controls, and definition of controls), two domains of comparability (study controls for the most important factor and any additional important factor), and three domains of exposure (ascertainment of exposure, same method of ascertainment for cases and controls, and the nonresponse rate). For cohort studies, quality is assessed for four domains of selection (representativeness of exposed cohort, selection of the nonexposed cohort, ascertainment of exposure, and demonstration that the outcome of interest was not present at start of study), two domains of comparability (study controls for the most important factor and any additional important factor), and three domains of outcome (method of assessment of outcome, follow-up period, and adequacy of follow-up of cohorts). Points are assigned based on specified levels of quality within each domain to a maximum of 9 points.

2.4. Statistical Analysis. Our a priori study protocol intention was to perform meta-analysis on eligible studies. Following the full-text review stage, we determined that pooling was not appropriate given the small number of studies and heterogeneity in methods; thus the studies were summarized qualitatively.

\section{Results}

3.1. Study Inclusion. A total of 962 unique publications were identified, of which 27 underwent full-text review, with 8 studies included in our summary [17-24] (Figure 1). Individual study characteristics are listed in Table 1.

We identified studies in RA $(n=4)$, SARDs $(n=2)$, and JIA $(n=2)$ populations; no studies were found for SLE, inflammatory myopathies, or scleroderma as unique entities. With the exception of studies from Sweden and Taiwan, all studies were of North American populations. One abstract each in the conditions of ANCA vasculitis [25] and Kawasaki Disease [26] was found, but they did not report risk estimates and thus were not included in the formal synthesis.

3.2. Rheumatoid Arthritis. Four studies included subjects with RA (two case-control studies $[18,19]$ and two cohort studies $[17,20])$ and examined associations with exposure to $\mathrm{NO}_{2}, \mathrm{SO}_{2}, \mathrm{PM}_{2.5}$, and $\mathrm{PM}_{10}$ (Table 2). In Hart et al., 2013, using data from the Nurses' Health Study and land use regression models, there was no definite evidence for increased RA risk related to a cumulative average exposure to $\mathrm{NO}_{2}, \mathrm{SO}_{2}$, $\mathrm{PM}_{10}$, or $\mathrm{PM}_{2.5}$ after adjustment for covariates [20]. In Hart et al., 2013, using data from the Swedish Epidemiological Investigation of Rheumatoid Arthritis study and land use regression models, the investigators were unable to demonstrate any increased risk for the development of RA with exposure to $\mathrm{NO}_{2}, \mathrm{PM}_{10}$, or $\mathrm{SO}_{2}$ [19]. In the study by De Roos et al., RA definitions were based on physician billing and prescription data; land use regression was used in the estimates for $\mathrm{PM}_{2.5}$ and $\mathrm{NO}_{2}$ as well as additional pollutants, black carbon and $\mathrm{NO}$, and the inverse distance weighting method was used for $\mathrm{PM}_{10}$ and $\mathrm{SO}_{2}$ estimates as well as for $\mathrm{NO}$, ozone, and $\mathrm{CO}$ [18]. When the RA definition required a specialist-confirmed 


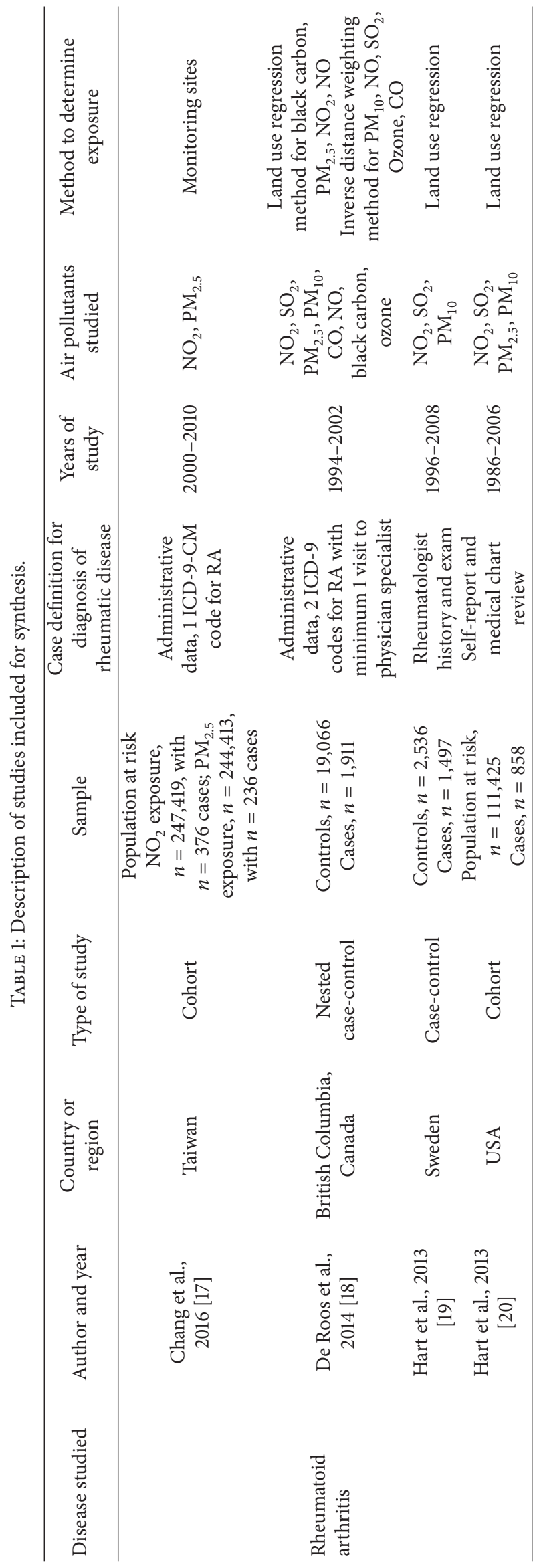




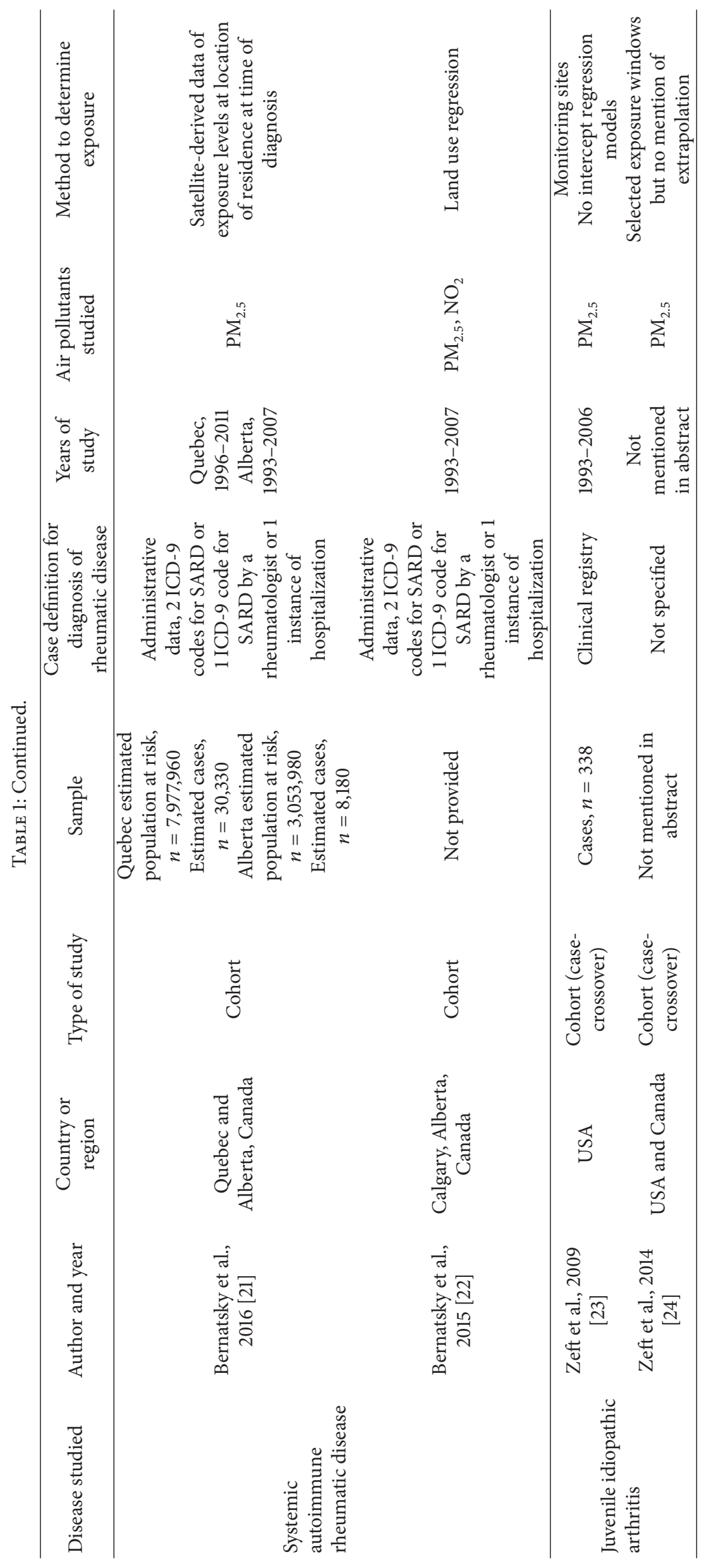




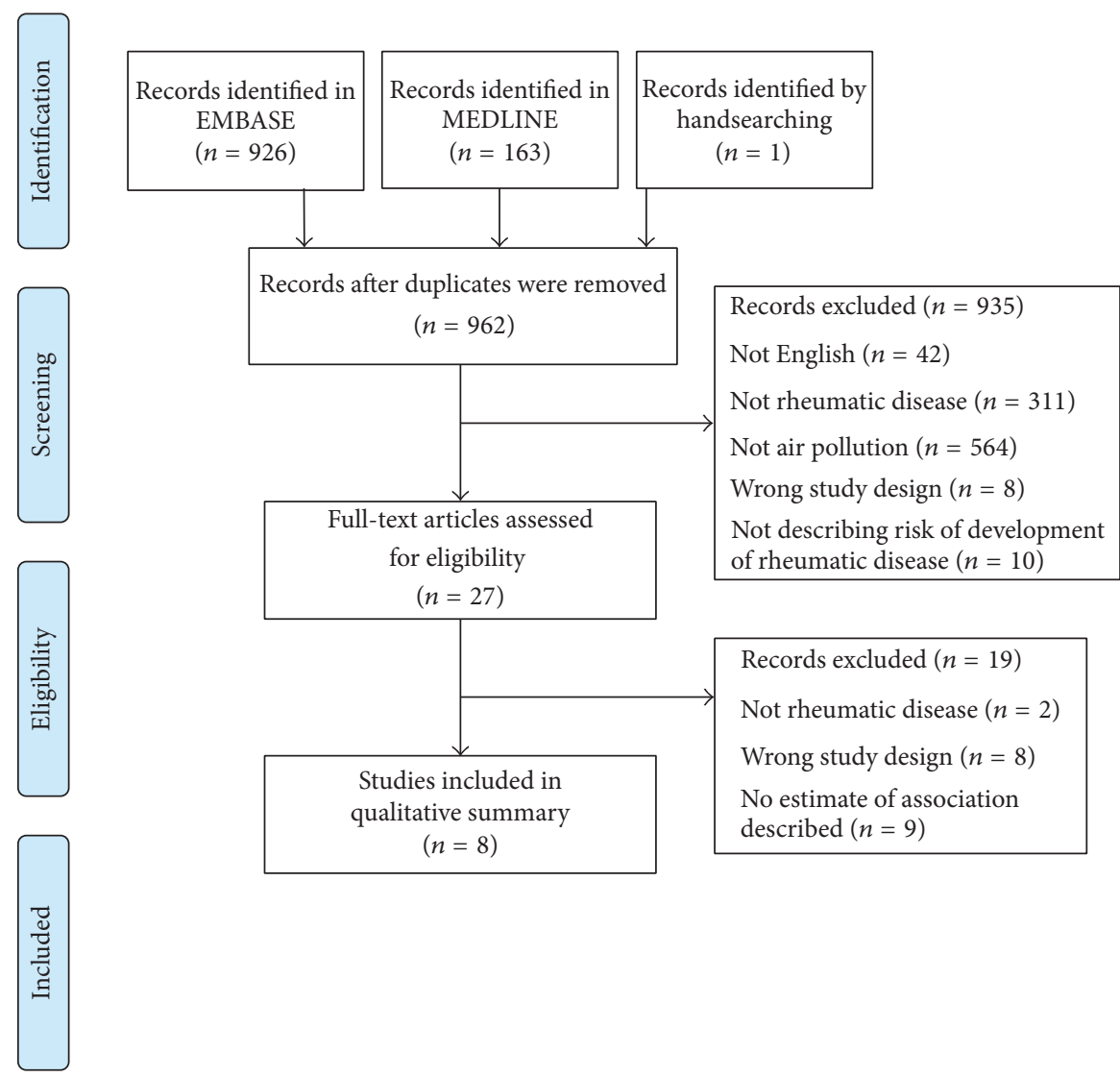

FIGURE 1: Study selection.

TABLE 2: Association between air pollutant exposure and the development of rheumatoid arthritis.

\begin{tabular}{|c|c|c|c|c|c|c|}
\hline Author & Study design & $\begin{array}{l}\text { Association } \\
\text { reported }\end{array}$ & $\begin{array}{l}\text { Nitrogen dioxide } \\
\qquad\left(\mathrm{NO}_{2}\right)\end{array}$ & $\begin{array}{c}\text { Fine particulate } \\
\text { matter }<2.5 \\
\text { microns }\left(\mathrm{PM}_{2.5}\right)\end{array}$ & $\begin{array}{c}\text { Fine particulate } \\
\text { matter }<10 \\
\text { microns }\left(\mathrm{PM}_{10}\right)\end{array}$ & $\begin{array}{l}\text { Sulfur dioxide } \\
\qquad\left(\mathrm{SO}_{2}\right)\end{array}$ \\
\hline Chang et al., 2016 [17] & Cohort & $\begin{array}{l}\mathrm{HR}^{*} \text { per } \\
\text { pollutant } \\
\text { level }^{* *}\end{array}$ & $\begin{array}{c}\text { Q2, } 1.12(95 \% \mathrm{CI}: \\
0.83 \text { to } 1.52) \text {; } \\
\text { Q3, } 1.53(95 \% \mathrm{CI}: \\
1.12 \text { to } 2.90) ; \\
\text { Q4, } 1.52(95 \% \mathrm{CI}: \\
1.11 \text { to } 2.08)\end{array}$ & $\begin{array}{c}\text { Q2, } 1.22(95 \% \mathrm{CI}: \\
0.85 \text { to } 1.74) ; \\
\text { Q3, } 1.15(95 \% \mathrm{CI}: \\
0.82 \text { to } 1.62) ; \\
\text { Q4, } 0.79(95 \% \mathrm{CI}: \\
0.53 \text { to } 1.16)\end{array}$ & Not reported & Not reported \\
\hline De Roos et al., 2014 [18] & $\begin{array}{c}\text { Nested } \\
\text { case-control }\end{array}$ & $\begin{array}{l}\text { OR per IQR } \\
\text { increase }^{* * *}\end{array}$ & $\begin{array}{c}0.90 \text { (95\% CI: } 0.85 \\
\text { to } 0.96)\end{array}$ & $\begin{array}{c}0.92 \text { (95\% CI: } 0.87 \\
\text { to } 0.98)\end{array}$ & $\begin{array}{c}0.91 \text { (95\% CI: } \\
0.86-0.96)\end{array}$ & $\begin{array}{c}0.88 \text { (95\% CI: } \\
0.82-0.93)\end{array}$ \\
\hline Hart et al., 2013 [19] & Case-control & $\begin{array}{c}\text { OR per IQR } \\
\text { increase over } \\
\text { average } \\
\text { exposure } \\
\text { ex*** }\end{array}$ & $\begin{array}{l}0.98 \text { (95\% CI: } 0.90 \\
\text { to } 1.07)\end{array}$ & Not reported & $\begin{array}{l}0.96 \text { (95\% CI: } 0.88 \\
\text { to } 1.04)\end{array}$ & $\begin{array}{c}1.01 \text { (95\% CI: } 0.93 \\
\text { to } 1.09)\end{array}$ \\
\hline Hart et al., 2013 [20] & Cohort & $\begin{array}{l}\text { HR per IQR } \\
\text { range } \\
\text { increase }^{* * * * *}\end{array}$ & $\begin{array}{c}0.92 \text { (95\% CI: } 0.85 \\
\text { to } 1.00)\end{array}$ & $\begin{array}{l}0.94 \text { (95\% CI: } 0.86 \\
\text { to } 1.04)\end{array}$ & $\begin{array}{l}0.92 \text { (95\% CI: } 0.85 \\
\text { to } 0.99)\end{array}$ & $\begin{array}{l}0.99 \text { (95\% CI: } 0.90 \\
\text { to } 1.09)\end{array}$ \\
\hline
\end{tabular}

HR: hazard ratio; IQR: interquartile range; OR: odds ratio.

${ }^{*}$ Adjusted for age, sex, urbanization level of residence, monthly income, and chronic obstructive pulmonary disease.

** $\mathrm{NO}_{2}$ : Quartile 1, <66,213 ppm (referent); Quartile 2, 66,213 to 86,908 ppm; Quartile 3, 86,099 to 99,882 ppm; Quartile 4, >99,992 ppm.

$\mathrm{PM}_{2.5}$ : Quartile 1, $<10,760 \mu \mathrm{m} / \mathrm{m}^{3}$ (referent); Quartile 2, 10,760 to $12,161 \mu \mathrm{m} / \mathrm{m}^{3}$; Quartile 3, 12,162 to $15,056 \mu \mathrm{m} / \mathrm{m}^{3}$; Quartile $4,>15,056 \mu \mathrm{m} / \mathrm{m}^{3}$.

*** Adjusted for age, sex, and neighborhood socioeconomic status.

***** Adjusted for age, sex, smoking status, and educational attainment.

***** Adjusted for age, race, smoking status and pack-years of smoking, age at menarche, parity, duration of lactation, menopause, hormone replacement therapy or oral contraceptive use, physical activity, body mass index, parental occupations, education, marital status, husband's education, family income, and house value. 
diagnosis, air pollutant exposure effect estimates were all inversely associated with the development of RA. In this study, residence proximity to roadway was additionally studied as a proxy for air pollutant exposure, with a significantly higher risk for RA for those within 50 metres from a highway compared to those over 150 metres away (OR: 1.37; 95\% CI: 1.11 to 1.68). In the study by Chang et al., data from monitoring sites were linked to administrative health data and incident RA cases were studied [17]. No association was found for $\mathrm{PM}_{2.5}$ exposure, but a significantly higher risk of incident $\mathrm{RA}$ was found in those exposed to the highest $\mathrm{NO}_{2}$ levels (adjusted HR for 3rd quartile: 1.53; 95\% CI: 1.12 to 2.09; adjusted HR for 4th quartile: 1.52; 95\% CI: 1.11 to 2.08).

3.3. Systemic Autoimmune Rheumatic Diseases. Bernatsky et al. reported the association between $\mathrm{PM}_{2.5}$ exposure and the odds of prevalent SARDs in case-control studies performed in Quebec and Alberta, Canada [21]. Exposure measurement was determined using average residential exposures at diagnosis based on satellite-derived data. In Alberta, a nonlinear association was found. The OR at $\mathrm{PM}_{2.5}$ exposures of 6.02 to $6.92 \mu \mathrm{g} / \mathrm{m}^{3}$ was 1.25 (95\% Credible Interval (CrI): 1.15 to 1.36), the OR at exposures of 6.92 to $8.11 \mu \mathrm{g} / \mathrm{m}^{3}$ was $1.03(95 \%$ CrI: 0.94 to 1.13 ), and the OR at exposures of $\geq 8.12 \mu \mathrm{g} / \mathrm{m}^{3}$ was 1.13 (95\% CrI: 1.02 to 1.25) after adjustment for sex, age, urban versus rural residence, and median income. In Quebec, increasing odds for increasing levels of $\mathrm{PM}_{2.5}$ exposure were demonstrated, with significant odds at levels of $\geq 11.81 \mu \mathrm{g} / \mathrm{m}^{3}$. In a study focused on one city in Alberta (Calgary) using land use regression models, exposure to $\mathrm{PM}_{2.5}$ appeared to be potentially associated with prevalent SARD (OR: 1.10; 95\% CrI: 1.01 to 1.22) in the model adjusted for sex, mean income, age $>45$ years, and interaction between age and sex [22]. No association with $\mathrm{NO}_{2}$ was demonstrated (OR: 1.02; 95\% CrI: 0.98 to 1.02$)$ [22].

3.4. Juvenile Idiopathic Arthritis. Two North American studies have explored the association between $\mathrm{PM}_{2.5}$ and JIA. From a patient population in Utah, 338 cases were identified based on a clinical examination by a rheumatologist. Exposure determination was based on monitoring sites data and no-intercept regression models. RR of 1.60 per $10 \mu \mathrm{g} / \mathrm{m}^{3}(95 \%$ CI: 1.00 to 2.54) for disease onset was found for children < 5.5 years of age but the results were imprecise when all ages were included in the analysis (RR: 1.11; 95\% CI: 0.85-1.45) [23]. The results were not replicated when studying a broader population in America and Canada with systemic-onset JIA [24].

3.5. Study Quality. The four studies in RA and two studies in SARDs were all deemed to be of high quality on the Newcastle-Ottawa scale in domains of selection, comparability, and exposure in the case-control studies and domains of selection, comparability, and outcome for the cohort study. Both studies in JIA were rated at lower quality, related to the case-crossover design selected. A summary of the quality assessment is found in Tables 3 and 4 .

\section{Discussion}

The goal of our research was to synthesize the published literature on associations between air pollution and the development of rheumatic disease. Air pollution has previously been associated with inflammation and other immunemediated diseases such as inflammatory bowel disease [2] and multiple sclerosis [3], with the hypothesis built on strong basic science and translational studies [6,27]. We identified relevant studies in RA, SARDs, and JIA conditions. In a cohort study from the USA and a case-control study from Sweden, no association between an increased RA risk and exposure to $\mathrm{NO}_{2}, \mathrm{SO}_{2}$, or $\mathrm{PM}$ was detected. In contrast, the cohort study from Taiwan found increased risk of RA with exposure to higher levels of $\mathrm{NO}_{2}$. Surprisingly, the casecontrol study by De Roos et al. did find an increased risk for RA based on proximity of the primary residence to highways but a potential reduced risk of developing RA in relation to air pollutant exposure [18], which is counterintuitive. In contrast, exposure to $\mathrm{PM}_{2.5}$ does appear to confer increased risk for SARDs and was a risk factor for JIA in US children below 5.5 years of age. We additionally identified abstracts on ANCA vasculitis [25] and Kawasaki Disease [26], which reported no association with exposure to $\mathrm{PM}_{10}$ and $\mathrm{PM}_{2.5}$, respectively, although estimates were not provided.

There are several possible reasons for the observed findings. Just as peak incidence of RA varies with age, there may be periods of life where the impact of air pollutants has greater influence on subsequent susceptibility to developing autoimmune diseases. Just as younger patients appeared to be more vulnerable to an association between air pollutants and JIA onset in Zeft et al's study [23], using multivariate analysis controlling for smoking, occupational exposure, home distance to sources of inhaled pollutants, seasonality, and traffic exposure, Orione et al. showed a significant association (odds ratio of 12.2) between carbon monoxide in the third trimester and the subsequent development of juvenile dermatomyositis [28]. Interactions between pollution exposure and specific risk alleles for different autoimmune conditions may also explain the difference in findings of association between air pollutants and different diseases.

Measurement of exposure is another important consideration when interpreting studies of pollution's effects on health. Largely, the studies employed place of residence prior to or at diagnosis to determine exposure, without accounting for places where leisure time, occupation, or daily commute might impact risk, resulting in exposure misclassification [29]. The measurement period, duration, and latency period between subclinical and clinical rheumatic diseases might result in wrongfully attributing exposure to the diagnosis period only. The varied composition of air pollution can make it challenging to overcome the confounding effects of concurrent pollutant exposure. Here distance to roadway studies have been conducted $[18,30]$, but further information on which pollutants create this heightened risk is required. Yet another consideration proposed is that the range and variability in pollution levels must be sufficiently large to detect associations, which may allow detection of risks limited to higher exposure levels [18]. 


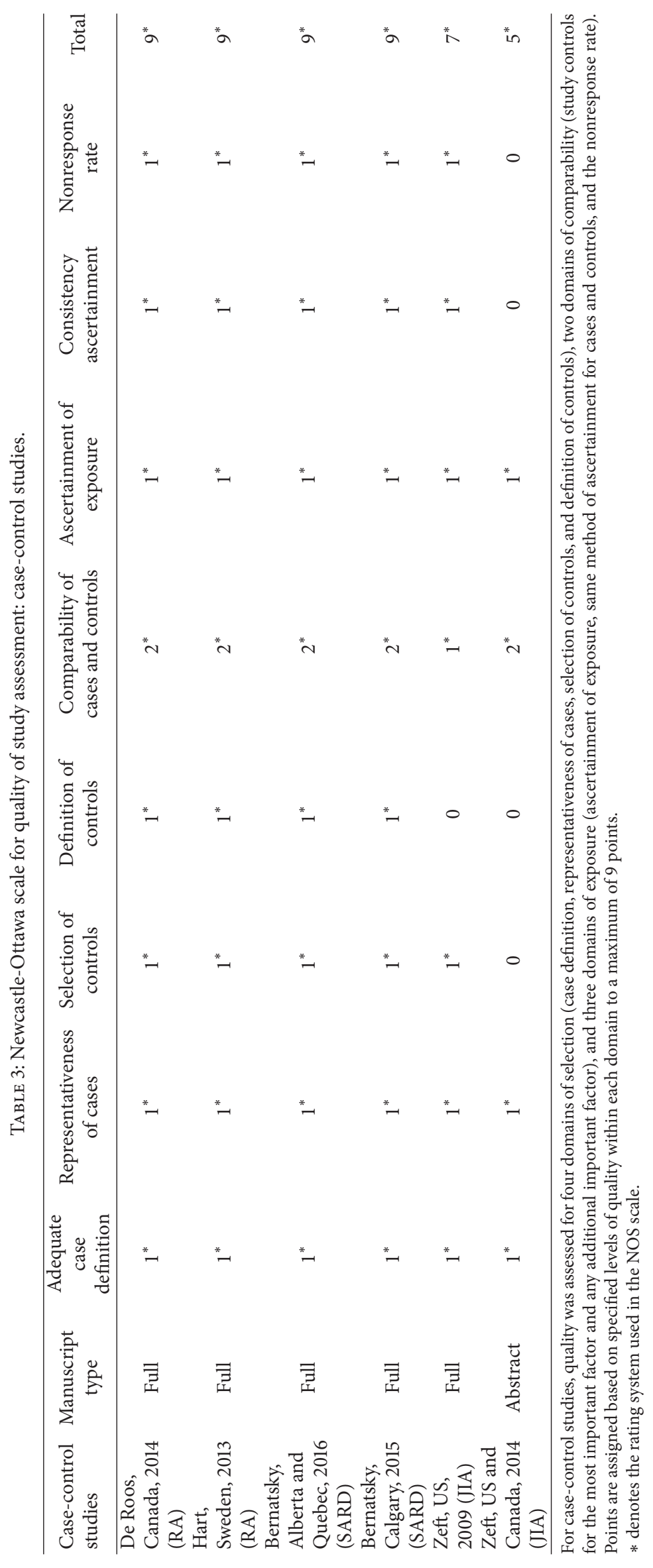




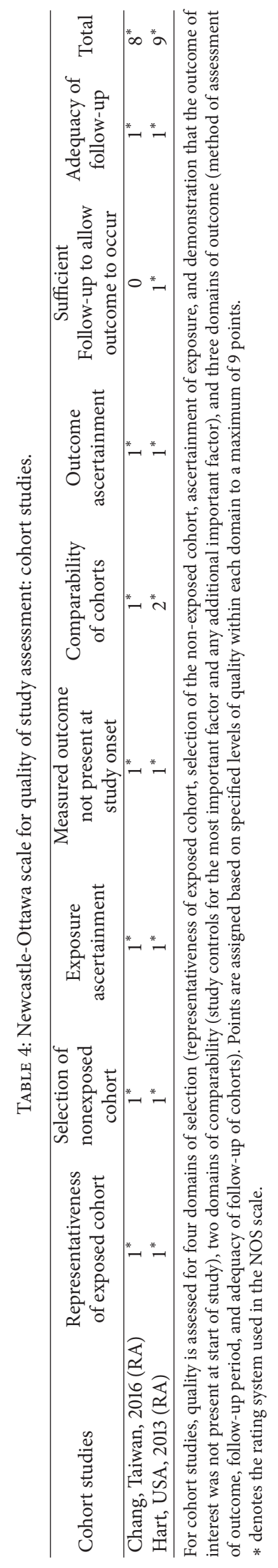


Our systematic review included a broad search strategy in order to ensure complete identification of relevant articles. We did not perform a meta-analysis because of the small number of eligible studies identified and their heterogeneity. Our systematic review serves as a valuable resource that highlights methodological considerations that should be considered in future research studies that explore the relationship between air pollution and immune-mediated diseases.

\section{Conclusion}

The existing studies suggest evidence for possible associations of $\mathrm{PM}_{2.5}$ exposure with SARDs development and JIA in younger age cohorts, but the evidence is less clear for links between air pollutant exposures and the development of RA. Additional epidemiologic work is suggested to improve upon existing analysis methods and expand studies of the effects of air pollution on disease phenotype and prognosis. More basic science and translational studies may also help to discover and explain the mechanisms behind progression from pollution related immune stimulation to the formation of antibodies and ultimately to progression of clinically apparent disease.

\section{Appendix}

\section{Search Strategy (Medline)}

(1) air pollution*/or air pollutant*/or air polluted/or air contamination*/or atmosphere pollution*/or atmosphere pollutant*/or atmosphere contamination*/or atmospheric pollution*/or atmospheric pollutant*or atmospheric contamination*/or "particulate matter"/ or " $\mathrm{PM}_{10}$ "/or " $\mathrm{PM}_{2.5}$ "/or ozone/or "O 3 "/or "carbon monoxide"/or carbonmonoxide/or "CO"/or "nitrogen dioxide"/or " $\mathrm{NO}_{2}$ "/or "sulphur dioxide"/or "sulphur dioxyde"/or "sulfurous anhydride"/or " $\mathrm{SO}_{2}$ ".ti,ab

(2) Air Pollution/or Particulate Matter/or Ozone/or Carbon Monoxide/or Nitrogen Dioxide/or Sulfur Dioxide.sh.

(3) $\mathrm{Or} /(1),(2)$

(4) Arthritis, Rheumatoid/

(5) rheumatoid arthritis.tw.

(6) exp Lupus Erythematosus, Systemic/

(7) systemic lupus erythematosus.tw.

(8) exp Arthritis, Juvenile/

(9) juvenile idiopathic arthritis.mp. or juvenile arthritis.tw. [mp = title, abstract, original title, name of substance word, subject heading word, keyword heading word, protocol supplementary concept word, rare disease supplementary concept word, unique identifier]

(10) exp Dermatomyositis/or exp Myositis/or exp Polymyositis/
(11) (dermatomyositis or inflammatory myo*).mp. or polymyositis.tw. [mp = title, abstract, original title, name of substance word, subject heading word, keyword heading word, protocol supplementary concept word, rare disease supplementary concept word, unique identifier]

(12) exp Scleroderma, Systemic/or exp Rheumatic Diseases/

(13) (SARD or systemic autoimmune rheumatic disease or scleroderma).mp. or systemic sclerosis.tw. [ $\mathrm{mp}=$ title, abstract, original title, name of substance word, subject heading word, keyword heading word, protocol supplementary concept word, rare disease supplementary concept word, unique identifier]

(14) exp Systemic Vasculitis/or exp Anti-Neutrophil Cytoplasmic Antibody-Associated Vasculitis/or exp Vasculitis/

(15) vasculitis.tw.

(16) (4) or (5) or (6) or (7) or (8) or (9) or (10) or (11) or (12) or (13) or (14) or (15)

(17) (3) and (16).

\section{List of Abbreviations}

RA: Rheumatoid arthritis

JIA: Juvenile idiopathic arthritis

SARDs: Systematic autoimmune rheumatic diseases

$\mathrm{PM}_{2.5}$ : $\quad$ Particulate matter $<2.5 \mu \mathrm{m}$ in size

$\mathrm{PM}_{10}$ : Particulate matter $<10 \mu \mathrm{m}$ in size

$\mathrm{SO}_{2}: \quad$ Sulfur dioxide

$\mathrm{NO}_{2}$ : Nitrogen dioxide

CO: $\quad$ Carbon monoxide

$\mathrm{O}_{3}$ : Ozone

SLE: $\quad$ Systemic lupus erythematosus

RR: $\quad$ Relative risk

HR: Hazard ratio

OR: Odds ratio

95\% CI: $95 \%$ confidence interval

95\% CrI: 95\% Credible Interval.

\section{Disclosure}

Dr. Barnabe is a Canadian Institutes for Health Research New Investigator in Community Based Primary Healthcare.

\section{Competing Interests}

The authors declare that they have no competing interests.

\section{Authors' Contributions}

Gavin Sun, Cheryl Barnabe, Gilaad G. Kaplan, and Sasha Bernatsky designed the study. Gavin Sun and Cheryl Barnabe performed study selection and data extraction. All authors contributed to interpretation of results, drafting of the manuscript, and approving the final manuscript. 


\section{Acknowledgments}

This work was supported by the Canadian Institutes for Health Research Team Grant: Challenges in Chronic Inflammation Initiative (PI Dr. B. Eksteen).

\section{References}

[1] A. Gibofsky, "Overview of epidemiology, pathophysiology, and diagnosis of rheumatoid arthritis," The American Journal of Managed Care, vol. 18, no. 13, pp. S295-S302, 2012.

[2] G. G. Kaplan, J. Hubbard, J. Korzenik et al., "The inflammatory bowel diseases and ambient air pollution: a novel association," The American Journal of Gastroenterology, vol. 105, no. 11, pp. 2412-2419, 2010.

[3] M. Oikonen, M. Laaksonen, P. Laippala et al., "Ambient air quality and occurrence of multiple sclerosis relapse," Neuroepidemiology, vol. 22, no. 1, pp. 95-99, 2003.

[4] D. Sugiyama, K. Nishimura, K. Tamaki et al., "Impact of smoking as a risk factor for developing rheumatoid arthritis: a metaanalysis of observational studies," Annals of the Rheumatic Diseases, vol. 69, no. 1, pp. 70-81, 2010.

[5] P. Stolt, H. Källberg, I. Lundberg, B. Sjögren, L. Klareskog, and L. Alfredsson, "Silica exposure is associated with increased risk of developing rheumatoid arthritis: Results from the Swedish EIRA Study," Annals of the Rheumatic Diseases, vol. 64, no. 4, pp. 582-586, 2005.

[6] H. Törnqvist, N. L. Mills, M. Gonzalez et al., "Persistent endothelial dysfunction in humans after diesel exhaust inhalation," American Journal of Respiratory and Critical Care Medicine, vol. 176, no. 4, pp. 395-400, 2007.

[7] S. Y. Salim, G. G. Kaplan, and K. L. Madsen, "Air pollution effects on the gut microbiota: a link between exposure and inflammatory disease," Gut Microbes, vol. 5, no. 2, pp. 215-219, 2014.

[8] R. Meier, W. E. Cascio, A. J. Ghio, P. Wild, B. Danuser, and M. Riediker, "Associations of short-term particle and noise exposures with markers of cardiovascular and respiratory health among highway maintenance workers," Environmental Health Perspectives, vol. 122, no. 7, pp. 726-732, 2014.

[9] J. Zhao, Z. Gao, Z. Tian et al., "The biological effects of individual-level $\mathrm{PM}_{2.5}$ exposure on systemic immunity and inflammatory response in traffic policemen," Occupational and Environmental Medicine, vol. 70, no. 6, pp. 426-431, 2013.

[10] M.-A. Bind, A. Baccarelli, A. Zanobetti et al., "Air pollution and markers of coagulation, inflammation, and endothelial function: associations and epigene-environment interactions in an elderly cohort," Epidemiology, vol. 23, no. 2, pp. 332-340, 2012.

[11] A. Hajat, M. Allison, A. V. Diez-Roux et al., "Long-term exposure to air pollution and markers of inflammation, coagulation, and endothelial activation a repeat-measures analysis in the multi-ethnic study of atherosclerosis (MESA)," Epidemiology, vol. 26, no. 3, pp. 310-320, 2015.

[12] S. B. Henderson, B. Beckerman, M. Jerrett, and M. Brauer, "Application of land use regression to estimate long-term concentrations of traffic-related nitrogen oxides and fine particulate matter," Environmental Science and Technology, vol. 41, no. 7, pp. 2422-2428, 2007.

[13] T. Bellander, N. Berglind, P. Gustavsson et al., "Using geographic information systems to assess individual historical exposure to air pollution from traffic and house heating in stockholm," Environmental Health Perspectives, vol. 109, no. 6, pp. 633-639, 2001.

[14] L. de Mesnard, "Pollution models and inverse distance weighting: some critical remarks," Computers and Geosciences, vol. 52, pp. 459-469, 2013.

[15] X. Song, Y. Liu, Y. Hu et al., "Short-term exposure to air pollution and cardiac arrhythmia: a meta-analysis and systematic review," International Journal of Environmental Research and Public Health, vol. 13, no. 7, article 642, 2016.

[16] The Newcastle-Ottawa Scale (NOS) for assessing the quality of nonrandomised studies in meta-analyses, http://www.ohri.ca/ programs/clinical_epidemiology/oxford.asp.

[17] K. H. Chang, C. C. Hsu, C. H. Muo et al., "Air pollution exposure increases the risk of rheumatoid arthritis: a longitudinal and nationwide study," Environment International, vol. 94, pp. 495499, 2016.

[18] A. J. De Roos, M. Koehoorn, L. Tamburic, H. W. Davies, and M. Brauer, "Proximity to traffic, ambient air pollution, and community noise in relation to incident rheumatoid arthritis," Environmental Health Perspectives, vol. 122, no. 10, pp. 1075-1080, 2014.

[19] J. E. Hart, H. Källberg, F. Laden et al., "Ambient air pollution exposures and risk of rheumatoid arthritis: results from the Swedish EIRA case-control study," Annals of the Rheumatic Diseases, vol. 72, no. 6, pp. 888-894, 2013.

[20] J. E. Hart, H. Källberg, F. Laden et al., "Ambient air pollution exposures and risk of rheumatoid arthritis," Arthritis Care and Research, vol. 65, no. 7, pp. 1190-1196, 2013.

[21] S. Bernatsky, A. Smargiassi, C. Barnabe et al., "Fine particulate air pollution and systemic autoimmune rheumatic disease in two Canadian provinces," Environmental Research, vol. 146, pp. 85-91, 2016.

[22] S. Bernatsky, A. Smargiassi, M. Johnson et al., "Fine particulate air pollution, nitrogen dioxide, and systemic autoimmune rheumatic disease in Calgary, Alberta," Environmental Research, vol. 140, pp. 474-478, 2015.

[23] A. S. Zeft, S. Prahalad, S. Lefevre et al., "Juvenile idiopathic arthritis and exposure to fine particulate air pollution," Clinical and Experimental Rheumatology, vol. 27, no. 5, pp. 877-884, 2009.

[24] A. S. Zeft, S. Prahalad, R. Schneider et al., "Systemic juvenile idiopathic arthritis and exposure to fine particulate air pollution," Arthritis and Rheumatology, vol. 66, article S136, 2014.

[25] R. Watts, D. Nelson, J. Mooney, A. MacGregor, and D. Scott, "Is there spatial clustering of ANCA-associated vasculitis in the UK?” Rheumatology, vol. 48, article i16, 2009.

[26] A. Zeft, J. Burns, R. Yeung et al., "A5.2 Kawasaki disease and exposure to fine particulate air pollution," Annals of the Rheumatic Diseases, vol. 74, article A47, 2015.

[27] R. Stiller-Winkler, H. Idel, G. Leng, C. Spix, and R. Dolgner, "Influence of air pollution on humoral immune response," Journal of Clinical Epidemiology, vol. 49, no. 5, pp. 527-534, 1996.

[28] M. A. M. Orione, C. A. Silva, A. M. E. Sallum et al., "Risk factors for juvenile dermatomyositis: exposure to tobacco and air pollutants during pregnancy," Arthritis Care and Research, vol. 66, no. 10, pp. 1571-1575, 2014. 
[29] N. A. Molodecky, R. Panaccione, S. Ghosh, H. W. Barkema, and G. G. Kaplan, "Challenges associated with identifying the environmental determinants of the inflammatory bowel diseases," Inflammatory Bowel Diseases, vol. 17, no. 8, pp. 1792-1799, 2011.

[30] J. E. Hart, F. Laden, R. C. Puett, K. H. Costenbader, and E. W. Karlson, "Exposure to traffic pollution and increased risk of rheumatoid arthritis," Environmental Health Perspectives, vol. 117, no. 7, pp. 1065-1069, 2009. 


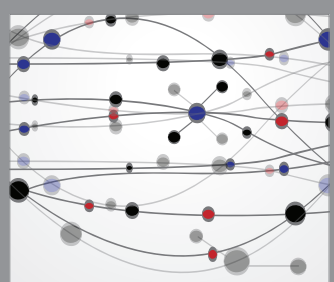

The Scientific World Journal
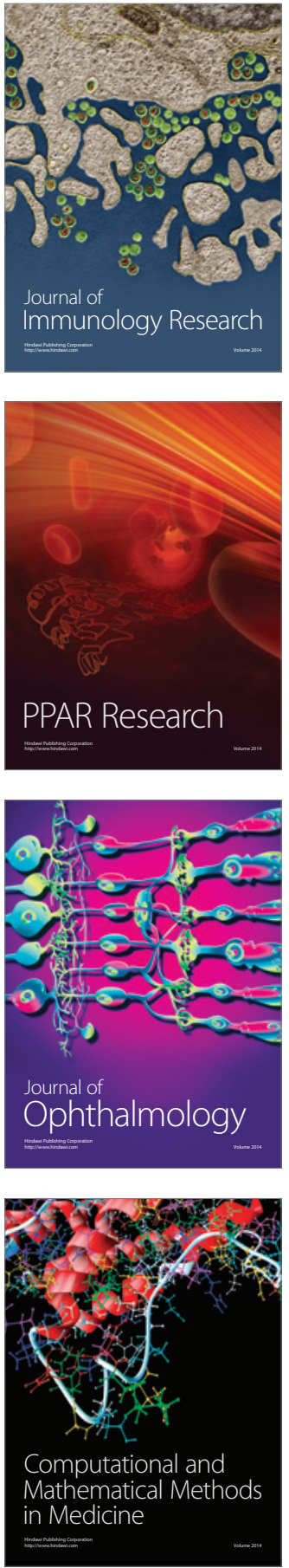

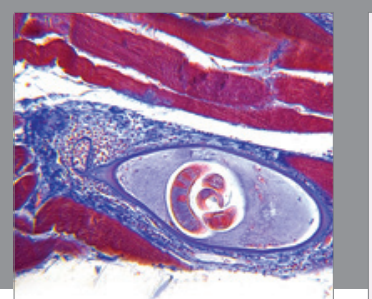

Gastroenterology Research and Practice

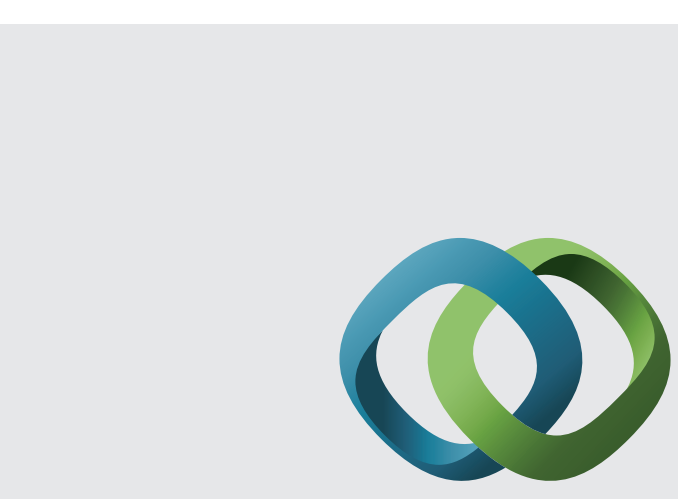

\section{Hindawi}

Submit your manuscripts at

http://www.hindawi.com
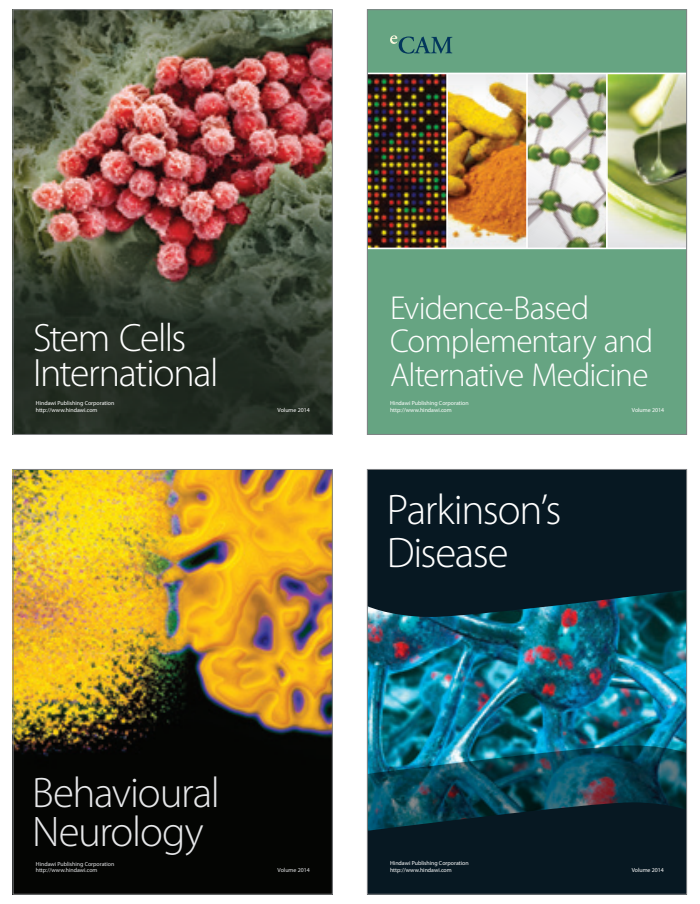
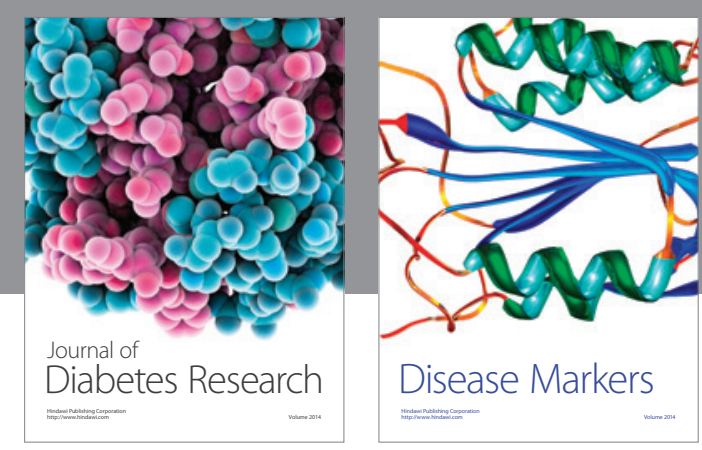

Disease Markers
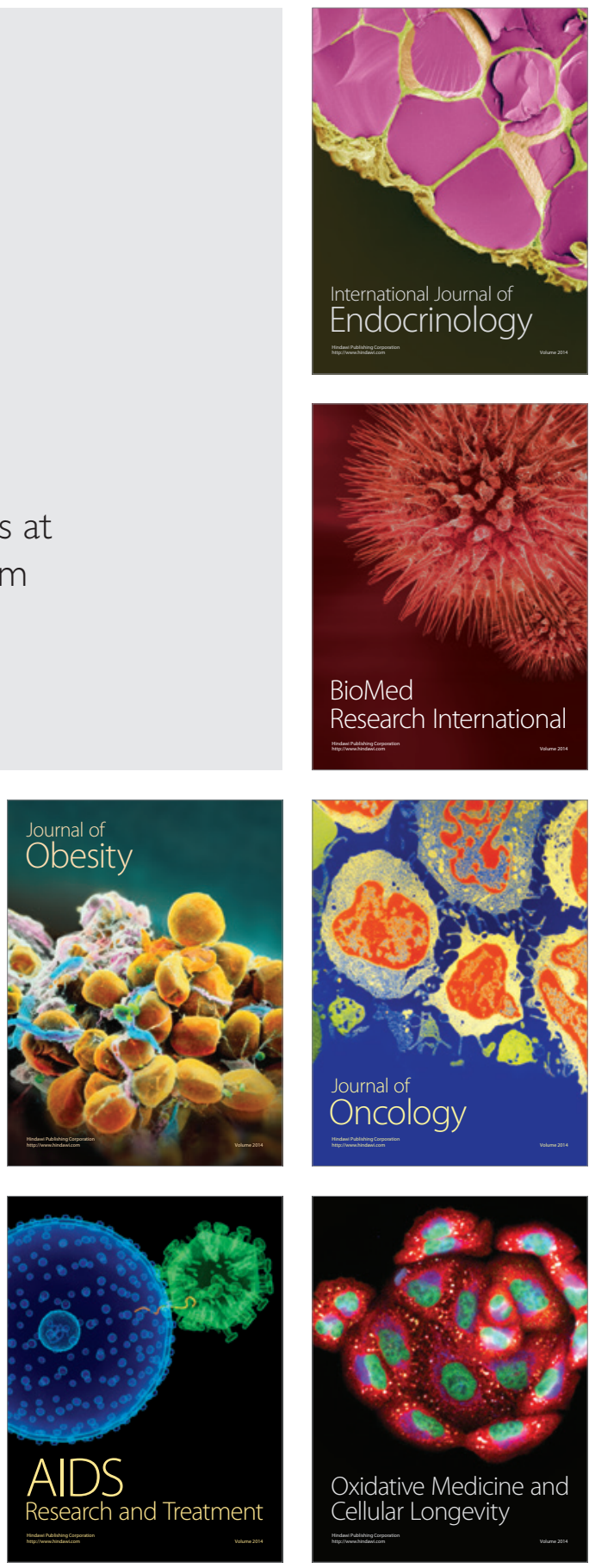